\title{
Prognostic value of neutrophil gelatinase-associated lipocalin in acute heart failure
}

\author{
Margarida Alvelos a,b,*, Patrícia Lourenço ${ }^{\mathrm{a}, \mathrm{b}}$, Carla Dias ${ }^{\mathrm{a}, \mathrm{b}}$, Marta Amorim ${ }^{\mathrm{a}, \mathrm{b}}$, Joana Rema ${ }^{\mathrm{a}, \mathrm{b}}$, \\ Ana Bento Leite ${ }^{\mathrm{a}, \mathrm{b}}$, João Tiago Guimarães ${ }^{\mathrm{c}, \mathrm{d}}$, Pedro Almeida ${ }^{\mathrm{e}}$, Paulo Bettencourt ${ }^{\mathrm{a}, \mathrm{b}}$ \\ a Department of Internal Medicine, Hospital São João, Porto, Portugal \\ b Department of Medicine, University of Porto Medical School, Porto, Portugal \\ c Department of Clinical Pathology, Hospital São João, Porto, Portugal \\ d Department of Biochemistry, University of Porto Medical School, Porto, Portugal \\ e Department of Cardiology, Hospital São João, Porto, Portugal
}

\section{A R T I C L E I N F O}

\section{Article history:}

Received 20 May 2011

Accepted 25 July 2011

Available online 27 August 2011

\section{Keywords:}

Acute heart failure

Neutrophil gelatinase-associated

lipocalin (NGAL)

Cystatin C

Prognosis

\begin{abstract}
A B S T R A C T
Background: The identification of patients at risk for worse outcome is still a challenge. We hypothesized that cystatin C, a marker of renal function, and neutrophil gelatinase-associated lipocalin (NGAL), a marker of acute renal injury, would have a role in the prognostic stratification of these patients.

Methods: We prospectively evaluated 121 patients admitted for acute HF. Serum NGAL and cystatin C levels were measured on the first morning after admission. The outcome measures used were the occurrence of death from all causes, and the combined endpoint defined as the first occurrence of either death or hospital admission. Patients were followed for up to 3 months.

Results: The variables associated with a higher occurrence of death in a univariate approach were older age and higher levels of BNP, cystatin C and NGAL, and those associated with the occurrence of the combined endpoint were older age, Diabetes mellitus, lower GFR, type 1 cardio-renal syndrome, BNP, cystatin C and NGAL. BNP and NGAL remained independent predictors of the occurrence of both all-cause death and the combined endpoint. NGAL levels in the 75 th percentile $(>167.5 \mathrm{ng} / \mathrm{mL})$ were associated with a 2.7-fold increase in the risk of death and a 2.9-fold increase in the risk of the first occurrence of either death or hospitalization.

Conclusions: Serum NGAL, a marker of acute renal injury, is an independent predictor of worse short term prognosis in patients with acute HF. This suggests a role of renal damage, apart from renal function, in the prognosis of these patients.
\end{abstract}

(c) 2011 Elsevier Ireland Ltd. All rights reserved.

\section{Introduction}

Acute heart failure (HF) is the most common cause of hospital admission in persons older than 65 years [1]. The prognosis of patients admitted for acute HF is dismal, with a mortality rate of $6,7 \%$ during hospitalization and $13,5 \%$ at 3 months $[2,3]$. The re-hospitalization rate remains very high, with $24 \%$ at 3 months and in the range of $30 \%$ to $50 \%$ during the first year $[3,4]$. The identification of patients at high risk of an adverse outcome is still a challenge to clinicians. A variety of predictors of ominous prognosis have been identified. Eleven to forty percent of the patients hospitalized for acute decompensated HF develop WRF during hospital stay [5] and WRF has been reported to

Abbreviations: HF, heart failure; WRF, worsening renal function; NGAL, neutrophil gelatinase-associated lipocalin; GFR, glomerular filtration rate.

is This investigation received a grant from "Fundação para a Ciência e a Tecnologia", project PIC/IC/82773/2007.

* Corresponding author at: Serviço de Medicina Interna, Hospital São João, Alameda Prof. Hernâni Monteiro, 4200-319 Porto, Portugal. Tel.: + 351 913238099; fax: + 351 225512332.

E-mail address: mpalvelos@gmail.com (M. Alvelos). be associated with higher in-hospital mortality, increased all-cause re-hospitalization rates and longer duration of hospital stay [6-9].

Cystatin $C$ is a cysteine protease inhibitor synthesized by nucleated cells that is freely filtered in the glomerulus, completely reabsorbed in the convoluted proximal tubule, and is not secreted. Cystatin $C$ levels are not affected by sex, age, race, or muscle mass. Previous reports suggested that cystatin $\mathrm{C}$ can have a role in prognosis stratification of acute HF patients [10-12].

Neutrophil gelatinase-associated lipocalin (NGAL) is a glycoprotein synthesized in the bone marrow during granulocyte maturation. Granulocytes, epithelial cells, renal tubular cells, and hepatocytes release NGAL during injury, and its levels are significantly elevated in epithelial damage [13-15]. Several reports have suggested that NGAL levels (both urinary and serum) are elevated in patients with acute kidney injury and type 1 cardio-renal syndrome and this rise in NGAL levels is known to precede the plasma creatinine increase [16-20]. We have previously reported that a single NGAL measurement independently predicts the development of type 1 cardio-renal syndrome in acutely decompensated HF patients, suggesting that it might be a useful biomarker in the early recognition of these patients [21]. 
Table 1

Baseline characteristics of patients as a function of death, and variables associated with all-cause death.

\begin{tabular}{|c|c|c|c|c|c|c|}
\hline & \multirow{2}{*}{$\begin{array}{c}\text { Total } \\
n=120\end{array}$} & \multicolumn{2}{|c|}{ Death } & \multirow[t]{2}{*}{$\mathrm{p}$} & \multirow[t]{2}{*}{$\mathrm{HR}(95 \% \mathrm{CI})$} & \multirow{2}{*}{$\begin{array}{c}\mathrm{p} \\
\text { value }\end{array}$} \\
\hline & & Yes $(n=27)$ & No $(n=93)$ & & & \\
\hline Age (years), media (SD); per year & $75.2( \pm 12.6)$ & $82.6( \pm 10.3)$ & $73.0( \pm 12.4)$ & $<0.001$ & $1.08(1.04-1.12)$ & $<0.001$ \\
\hline Male sex, n (\%) & $61(50.8)$ & $12(44.4)$ & $49(52.7)$ & 0.59 & $0.79(0.37-1.69)$ & 0.55 \\
\hline Diabetes mellitus, n (\%) & $30(25.0)$ & $6(22.2)$ & $24(25.8)$ & 0.90 & $0.87(0.35-2.16)$ & 0.76 \\
\hline LVEF (\%), median (IQR); per $1 \%$ & $35(25-45)$ & $35(29-46)$ & $35(24-45)$ & 0.59 & $1.01(0.97-1.04)$ & 0.70 \\
\hline LVEF< $45 \%$ (vs. HFPEF), n (\%) & $80(67.2)$ & $18(69.2)$ & $62(66.7)$ & 0.99 & $1.16(0.51-2.68)$ & 0.72 \\
\hline NYHA IV (vs. III), n (\%) & $80(66.7)$ & $21(77.8)$ & $59(63.4)$ & 0.25 & $1.93(0.78-4.79)$ & 0.16 \\
\hline Systolic blood pressure ( $\mathrm{mm} \mathrm{Hg}$ ), media (SD); per mm Hg & $123( \pm 28)$ & $114( \pm 21)$ & $125( \pm 29)$ & 0.06 & $0.99(0.97-1.00)$ & 0.07 \\
\hline Heart rate (bpm), media (SD); per bpm & $85( \pm 20)$ & $85( \pm 17)$ & $85( \pm 21)$ & 0.82 & $1.00(0.98-1.02)$ & 0.86 \\
\hline Diuretic at admission, $\mathrm{n}(\%)$ & $106(88.3)$ & $26(96.3)$ & $80(86.0)$ & 0.19 & $3.79(0.51-27.94)$ & 0.19 \\
\hline Diuretic dose at admission (mg/day), median (IQR); per mg/day & $80(60-100)$ & $80(60-100)$ & $80(60-100)$ & 0.42 & $1.00(0.99-1.02)$ & 0.40 \\
\hline ACEi or ARB, n (\%) & $81(67.5)$ & $17(63.0)$ & $64(68.8)$ & 0.74 & $0.82(0.38-1.80)$ & 0.62 \\
\hline Beta-blocker, n (\%) & $61(50.8)$ & $12(44.4)$ & $49(52.7)$ & 0.59 & $0.76(0.36-1.63)$ & 0.48 \\
\hline GFR $\left(\mathrm{mL} / \mathrm{min}\right.$ per $\left.1.73 \mathrm{~m}^{2}\right)$, median (IQR); per $\mathrm{mL} / \mathrm{min}$ & $40.0( \pm 16.5)$ & $36.6( \pm 15.4)$ & $41.3( \pm 16.7)$ & 0.20 & $0.98(0.96-1.01)$ & 0.16 \\
\hline CRS1, n (\%) & $22(18.3)$ & $8(29.6)$ & $14(15.1)$ & 0.15 & $2.23(0.98-5.09)$ & 0.06 \\
\hline BNP (pg/mL), median (IQR); per 100 pg/mL & $1594(773.0-2702.0)$ & $2496(995.0-3785.0)$ & $1368(689.4-2285.5)$ & 0.004 & $1.18(1.08-1.30)$ & $<0.001$ \\
\hline Cystatin C (mg/L), media (SD); 75th percentile vs. others & $1.70(0.79)$ & $2.00(0.96)$ & $1.59(0.69)$ & 0.02 & $1.76(1.16-2.66)$ & 0.008 \\
\hline NGAL (ng/mL), median (IQR); 75th percentile vs. others & $95.0(62.0-167.5)$ & $98.0(77.0-213.0)$ & $91.0(60.0-147.0)$ & 0.08 & $1.04(1.02-1.06)$ & $<0.001$ \\
\hline
\end{tabular}

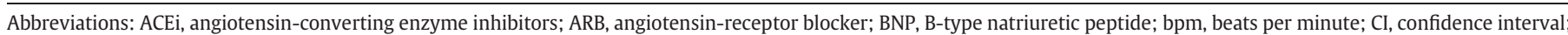

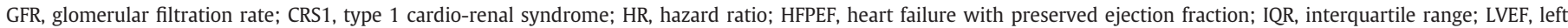
ventricular ejection fraction; NGAL, neutrophil gelatinase-associated lipocalin; NYHA, New York Heart Association; SD, standard deviation.

There is an increasing body of evidence supporting the prognostic impact of several markers of tubulo-interstitial damage, including NGAL, in various renal disorders [14,22-24]. In HF, contrariwise, the prognostic value of these markers has not been well established. In a recent study, both urinary kidney injury molecule-1 and N-acetyl$\beta$-(D)-glucosaminidase, but not NGAL, showed to add prognostic information to glomerular filtration rate (GFR) in chronic HF patients [25], suggesting an important role for tubular damage in cardio-renal interaction in HF. Recently, higher serum NGAL levels were reported to be associated with poorer 2-year survival in a chronic HF population [26].

We aimed to evaluate the short term prognostic significance of biomarkers of renal function and injury in acute HF patients.

\section{Materials and methods}

2.1. Study sample

We prospectively studied 121 patients admitted to our Internal Medicine Department between May and November 2009 with the diagnosis of acute HF. Patients were eligible whether acute HF was de novo or an exacerbation of chronic HF symptoms with an increase in at least one New York Heart Association class. HF diagnosis was based on the European Society of Cardiology criteria. Patients with an acute coronary syndrome and patients on chronic renal function replacement therapy were excluded.

The study protocol conformed the Declaration of Helsinki, local ethics committee approved the study and patients gave informed consent.

\subsection{Study design}

Fasting venous blood samples were collected between 8:00 and 9:00a.m. on the first morning after admission. NGAL and cystatin C levels were measured on the first morning after admission. Specimens were centrifuged for $10 \mathrm{~min}$ at $3000 \times \mathrm{g}$ within $2 \mathrm{~h}$ after laboratory arrival. Analytical parameters were measured at the Hospital de São João Clinical Pathology Department. NGAL measurement was made with the Triage NGAL test system using EDTA-anticoagulated whole blood. This test system is a rapid, point-of-care fluorescence detection immunoassay using the Triage meter (Biosite, Quilaban, Lisboa, Portugal). Several drops of blood are added to the sample port in the device. After addition of the sample, the blood cells are separated from the plasma using a filter contained in the test device. The results are displayed in approximately $15 \mathrm{~min}$. The manufacturer provided the calibration curve. For each 24 patient samples in which NGAL was determined, one control was performed. The lowest detectable concentration is $60 \mathrm{ng} / \mathrm{mL}$, and the test has been demonstrated to be linear from 60 to $1300 \mathrm{ng} / \mathrm{mL}$ NGAL (which is considered the measurable range). We found a within-run precision of $19.3 \%$ for a sample with an average $132 \mathrm{ng} / \mathrm{mL}$. Serum cystatin C was assayed using a particle-enhanced immunonephelometric assay ( $\mathrm{N}$ Latex Cystatin $\mathrm{C}$,

Table 2

Baseline characteristics of patients as a function of death or hospitalization, and variables associated with death or hospitalization.

\begin{tabular}{|c|c|c|c|c|c|}
\hline & \multicolumn{2}{|c|}{ Death or hospitalization } & \multirow[t]{2}{*}{$\mathrm{p}$} & \multirow[t]{2}{*}{$\mathrm{HR}(95 \% \mathrm{CI})$} & \multirow{2}{*}{$\begin{array}{c}\mathrm{p} \\
\text { value }\end{array}$} \\
\hline & Yes $(n=53)$ & No $(n=67)$ & & & \\
\hline Age (years), media (SD); per year & $77.8( \pm 12.0)$ & $73.1( \pm 12.8)$ & 0.04 & $1.03(1.00-1.05)$ & 0.04 \\
\hline Male sex, n (\%) & $27(50.9)$ & $34(50.7)$ & 1.00 & $1.05(0.61-1.80)$ & 0.86 \\
\hline Diabetes mellitus, n (\%) & $18(34)$ & $12(17.9)$ & 0.07 & $2.00(1.13-3.54)$ & 0.02 \\
\hline LVEF (\%), median (IQR); per $1 \%$ & $38(27-45)$ & $35(23-45)$ & 0.42 & $1.01(0.98-1.03)$ & 0.54 \\
\hline LVEF $<45 \%$ (vs. HFPEF), n (\%) & $35(67.3)$ & $45(67.2)$ & 1.00 & $1.03(0.58-1.83)$ & 0.93 \\
\hline NYHA IV (vs. III), n (\%) & $38(71.7)$ & $42(62.7)$ & 0.40 & $1.43(0.79-2.60)$ & 0.24 \\
\hline Systolic blood pressure ( $\mathrm{mm} \mathrm{Hg}$ ), media (SD); per mm Hg & $121( \pm 26)$ & $124( \pm 29)$ & 0.51 & $1.00(0.98-1.01)$ & 0.84 \\
\hline Heart rate (bpm), media (SD); per bpm & $83( \pm 18)$ & $87( \pm 22)$ & 0.27 & $0.99(0.98-1.01)$ & 0.29 \\
\hline Diuretic at admission, $\mathrm{n}(\%)$ & $50(94.3)$ & $56(83.6)$ & 0.09 & $2.75(0.86-8.83)$ & 0.09 \\
\hline Diuretic dose at admission (mg/day), median (IQR); per mg/day & $80(60-100)$ & $80(60-100)$ & 0.40 & $1.00(1.00-1.01)$ & 0.29 \\
\hline ACEi or ARB, n (\%) & $35(66.0)$ & $46(68.7)$ & 0.91 & $0.95(0.54-1.68)$ & 0.86 \\
\hline Beta-blocker, n (\%) & $23(43.4)$ & $38(56.7)$ & 0.21 & $0.68(0.39-1.16)$ & 0.16 \\
\hline GFR $\left(\mathrm{mL} / \mathrm{min}\right.$ per $\left.1.73 \mathrm{~m}^{2}\right)$, median (IQR); per $\mathrm{mL} / \mathrm{min}$ & $35.7( \pm 15.4)$ & $43.8( \pm 16.6)$ & 0.008 & $0.98(0.96-0.99)$ & 0.006 \\
\hline CRS1, n (\%) & $14(26.4)$ & $8(11.9)$ & 0.07 & $1.99(1.08-3.67)$ & 0.03 \\
\hline BNP (pg/mL), median (IQR); per 100 pg/mL & $1797(916.5-3233.0)$ & $1277(626.0-2268.0)$ & 0.04 & $1.12(1.04-1.21)$ & 0.005 \\
\hline Cystatin C (mg/L), media (SD); 75th percentile vs. others & $1.91(0.84)$ & $1.50(0.66)$ & 0.004 & $1.66(1.22-2.26)$ & 0.001 \\
\hline NGAL (ng/mL), median (IQR); 75th percentile vs. others & $102.0(76.0-202.0)$ & $84.0(60.0-139.0)$ & 0.02 & $1.04(1.02-1.05)$ & $<0.001$ \\
\hline
\end{tabular}

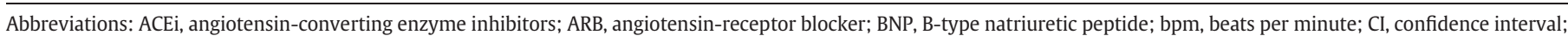

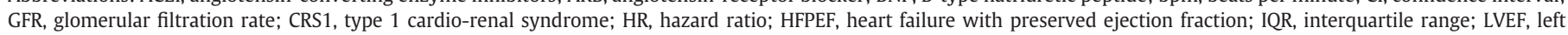
ventricular ejection fraction; NGAL, neutrophil gelatinase-associated lipocalin; NYHA, New York Heart Association; SD, standard deviation. 

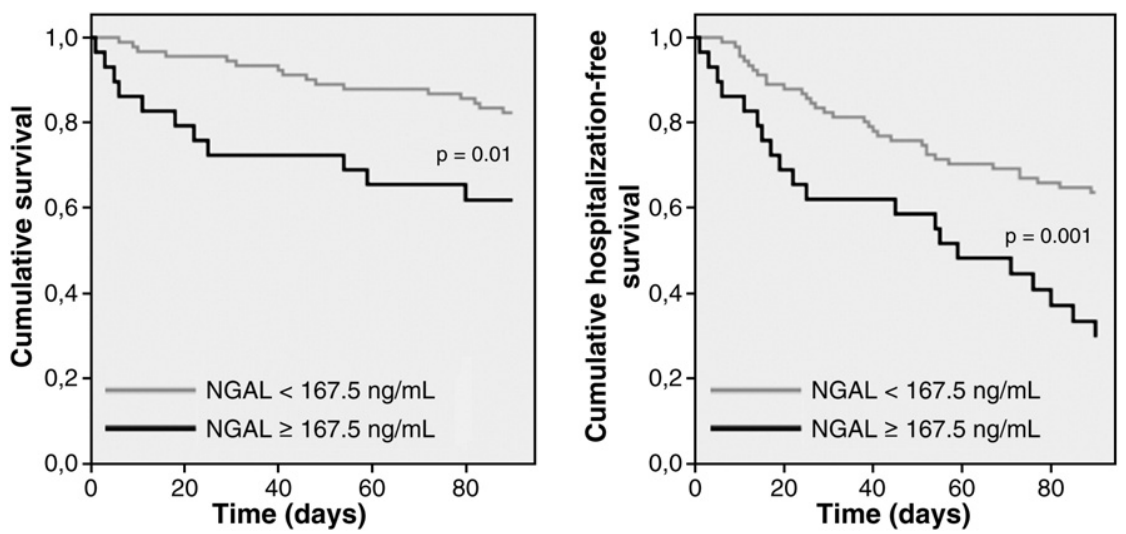

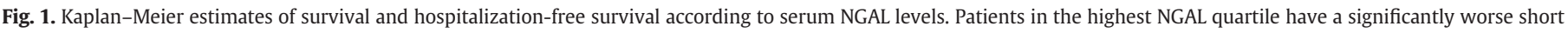
term prognosis than the ones in the other quartiles.

Siemens, Lisboa, Portugal) on a BN II laser nephelometer. The lower limit of detection is $0.05 \mathrm{mg} / \mathrm{L}$, and the within-run and the run-to-run variation was $<5 \%$. Plasma B-type natriuretic peptide was measured using an Architect i2000 automated analyzer (Abbott, Lisboa, Portugal). Serum creatinine, urea and albumin were measured using conventional methods with an Olympus AU5400 automated clinical chemistry analyzer. (Beckman-Coulter, Izasa, Porto, Portugal). Blood counts were obtained using an automated blood counter (Sysmex XE-5000; Emílio de Azevedo Campos, Porto, Portugal). GFR was estimated according to the Modification of Diet in Rena Disease (MDRD) formula: GFR $=170 \times[\mathrm{PCr}]^{-0.999} \times[\mathrm{Age}]^{-0.176} \times[\mathrm{PU}]^{-0.170} \times[$ Albu$\mathrm{min}]^{+0.318} \times[0.762$ if female $] \times[1.180$ if black $]$ [27-29]. All patients performed an echocardiogram and the left ventricular ejection fraction was determined using the modified Simpson method. Left ventricular systolic function was considered preserved if the left ventricular ejection fraction was $\geq 45 \%$. Patients received standard treatment according to the attending physicians. Physicians were blinded to NGAL and cystatin C levels. Type 1 cardio-renal syndrome was defined as an increase in the creatinine level of at least $0.3 \mathrm{mg} / \mathrm{dL}$ or $50 \%$ of basal creatinine during hospitalization.

The outcome measures used to determine the prognostic importance of serum NGAL and cystatin C were the occurrence of death from all causes, and the combined endpoint defined as the first occurrence of either death or hospital admission. Information about the outcomes was obtained searching on our hospital records and by telephone contact with the patients. Patients were followed for up to 3 months after the first day of hospitalization. One patient was lost to follow-up.

\subsection{Statistical analysis}

Numerical variables are presented as mean (standard deviation) if normally distributed or median (interquartile range) if non-normally distributed. Categorical variables are presented as count (percent). Patients who developed the study outcomes and those who did not were compared: the $\chi^{2}$ test was used to compare categorical variables, a two independent-sample $t$ test was used to compare normally distributed variables, and the Mann-Whitney $U$ test was used for skewed variables. We used Kaplan-Meier curves to display survival and hospitalization-free survival depending on serum NGAL and cystatin C levels. The study sample was categorized according to the 75th percentile of NGAL and cystatin C: $167.5 \mathrm{ng} / \mathrm{mL}$ and $1.98 \mathrm{mg} / \mathrm{L}$, respectively. BNP was analyzed per $100 \mathrm{pg} / \mathrm{mL}$ increases. A Cox regression analysis was used to assess which variables predicted both the isolated and the combined endpoints. A multivariate model was built based on the variables associated with the outcome in the univariate approach. All of the analyses were conducted using SPSS 18.0 (SPSS, Inc., Chicago, IL). $\mathrm{p}<0.05$ was considered to be statistically significant.

\section{Results}

Clinical characteristics of the study patients are presented in Table 1. A comparison between those who developed the study outcomes and those who did not are presented in Tables 1 and 2. At 90 days 27 (22.5\%) patients died and 53 (44.2\%) met the combined endpoint of death or hospital admission. Patients who died were older and presented higher levels of plasma BNP and serum cystatin $C$ at hospital admission. There was a trend for lower systolic blood pressure measurements and higher levels of serum NGAL in this group. Patients who met the combined endpoint of death or hospitalization were also older, had lower GFRs and higher levels of BNP, cystatin C and NGAL. There was a propensity for having a prior diagnosis of Diabetes mellitus, for being under diuretics at the time of hospital admission and for developing type 1 cardio-renal syndrome during hospitalization.

The variables associated with a higher occurrence of death in the univariate analysis were older age and higher levels of BNP, cystatin C and NGAL. Patients developing type 1 cardio-renal syndrome presented a higher risk of death, nevertheless it did not reach statistical significance. Similarly, the variables associated with the occurrence of the combined endpoint of death or hospitalization were older age, the presence of Diabetes mellitus, lower GFR, type 1 cardio-renal syndrome, BNP, cystatin C and NGAL.
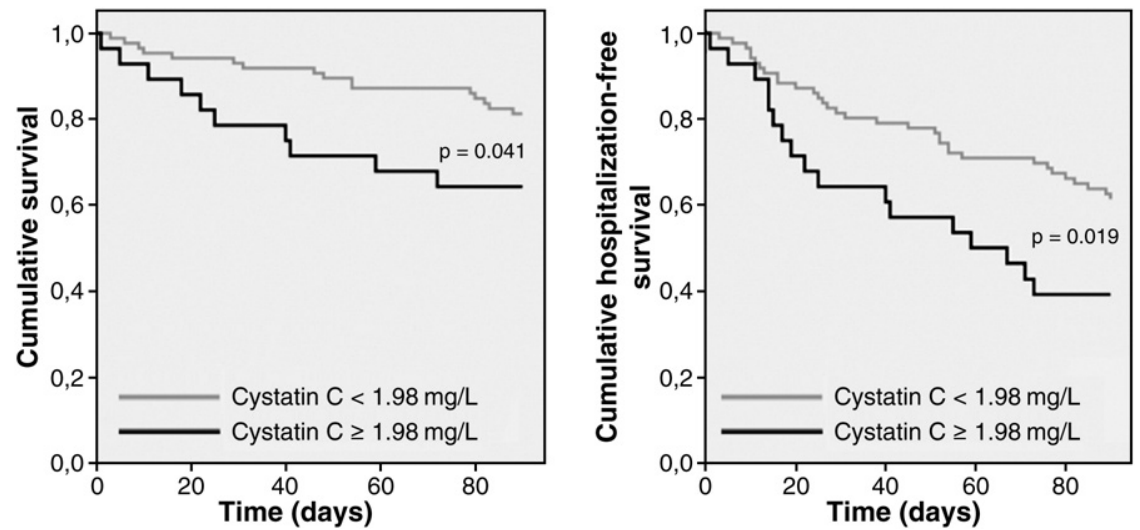

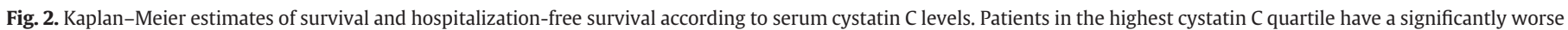
short term prognosis than the ones in the other quartiles. 
Table 3

Multivariate model for prediction of all-cause death.

\begin{tabular}{lcc}
\hline & \multicolumn{2}{c}{ Death } \\
\cline { 2 - 3 } & HR $(95 \% \mathrm{CI})$ & p value \\
\hline Age, per year & $1.065(1.025-1.107)$ & 0.001 \\
BNP, per $100 \mathrm{pg} / \mathrm{mL}$ & $1.017(1.006-1.027)$ & 0.002 \\
NGAL, 75th percentile vs. others & $2.696(1.203-6.041)$ & 0.016 \\
CRS1 & & 0.339 \\
Cystatin C, 75th percentile vs. others & & 0.771 \\
\hline
\end{tabular}

Abbreviations: BNP, B-type natriuretic peptide; CI, confidence interval; CRS1, type 1 cardio-renal syndrome; HR, hazard ratio; NGAL, neutrophil gelatinase-associated lipocalin.

Kaplan-Meier curves show that patients with baseline serum NGAL above the 75 th percentile $(167.5 \mathrm{ng} / \mathrm{mL})$ had a significantly higher 3-month mortality and higher occurrence of the combined endpoint of death or hospitalization (Fig. 1) than the patients with lower NGAL levels. Similarly, patients with baseline serum cystatin C above the 75 th percentile $(1.98 \mathrm{mg} / \mathrm{L})$ also performed worse within the 3-month follow-up period, weather considering the isolated or the combined endpoint (Fig. 2).

Tables 3 and 4 show the multivariate models built. Older age and higher levels of BNP and NGAL were independently associated with a higher occurrence of death. Higher levels of BNP and NGAL were also independent predictors of the occurrence of the combined outcome of death or hospitalization. NGAL levels in the 75th percentile $(>167.5 \mathrm{ng} / \mathrm{mL})$ were associated with a 2.7 -fold increase in the 3-month risk of death and a 2.9-fold increase in the 3-month risk of the first occurrence of either death or hospitalization.

\section{Discussion}

In our study sample, serum NGAL was an independent predictor of worse short term prognosis, namely of the occurrence of death or the combined outcome of death or hospitalization, when measured at admission in patients hospitalized for acute HF. Serum NGAL levels above $167.5 \mathrm{ng} / \mathrm{mL}$ predicted an almost 3-fold increase in morbidity and mortality within 3 months after admission for acute HF. The interplay between heart and kidney in HF has gathered growing interest in recent years and has been subject of intense and increasing clinical investigation. The actual knowledge emphasizes the role of the damaged heart signaling the kidneys through hemodynamic factors [30]. However, it is recognized that this interaction is most probably bidirectional. Other factors of the damaged heart contributing to kidneys damage as well as factors from the damaged kidney acting in the heart are currently under investigation.

NGAL is a biomarker reflecting damage to renal tubular cells, whose urinary and serum levels have been shown to be highly increased in patients with acute and chronic renal injury in different stages and settings [14,16-20,22-24,31,32]. NGAL levels rise in urine and serum as early as from $2 \mathrm{~h}$ onwards after an acute insult to the kidneys [16-20], and they can help predicting early type 1 cardio-renal syndrome development in patients admitted with worsening HF [21].

In the present report of 121 patients with acute HF, NGAL strongly predicted higher short term morbidity and mortality. This predictive power was independent of other variables also associated with the outcome, namely indexes of baseline renal function. In several previous reports the prognostic value of WRF in acute HF has been consistently shown and this has been true whether renal function is evaluated based on plasma creatinine, GFR or cystatin C [[],[]]. In our population, although renal function, as measured by MDRD and cystatin C, showed prognostic value in an univariate approach, in the multivariate model built, only NGAL independently predicted worse outcome, suggesting a role of renal injury, besides that of renal function and independent of it, upon the beginning of acute HF episodes.
Table 4

Multivariate model for prediction of death or hospitalization.

\begin{tabular}{lcr}
\hline & \multicolumn{2}{c}{ Death or hospitalization } \\
\cline { 2 - 3 } & HR $(95 \% \mathrm{CI})$ & p value \\
\hline BNP, per $100 \mathrm{pg} / \mathrm{mL}$ & $1.014(1.005-1.022)$ & 0.002 \\
NGAL, 75th percentile vs. others & $2.860(1.593-5.136)$ & $<0.001$ \\
Age, per year & 0.153 \\
Diabetes mellitus & 0.061 \\
GFR, per mL/min & 0.360 \\
CRS1 & 0.257 \\
Cystatin C, 75th percentile vs. others & 0.621 \\
\hline
\end{tabular}

Abbreviations: BNP, B-type natriuretic peptide; CI, confidence interval; GFR, glomerular filtration rate; CRS1, type 1 cardio-renal syndrome; HR, hazard ratio; NGAL, neutrophil gelatinase-associated lipocalin.

Moreover, as far as we could understand from literature review, these are the first data on the prognostic impact of NGAL levels in an acute HF setting, thus broadening its predictive properties beyond the spectrum of renal disease.

Limitations of our study include the relatively small sample size and its single-centered nature. The observational design precluded the evaluation of the possible prognostic implications of clinical management changes based on admission NGAL levels. This was not, however, an objective of the study. Our results do not clarify if the increase in NGAL results from acute kidney injury or if it is associated with tubulo-interstitial chronic injury, as suggested by the observed increase in urinary NGAL in chronic HF with worse outcome [26]. Nevertheless, this study was able to test our hypothesis and to detect an independent association of increased NGAL levels with worse outcomes in acute HF patients, suggesting a role of renal damage, apart from renal function, in the prognosis of patients with a failing heart. Algorithms for risk stratification of acute HF patients would probably be enriched if renal injury measurements, namely NGAL, were included.

\section{Conclusions}

High serum NGAL independently predicts worse short term prognosis in patients with acute $\mathrm{HF}$, with levels in the highest quartile, above $167.5 \mathrm{ng} / \mathrm{mL}$, forecasting an almost 3-fold increase in morbidity and mortality within 3 months after admission. These results suggest that, in acute HF, renal injury, beyond renal function, has a relevant role in the interaction between heart and kidney.

\section{Acknowledgment}

The authors of this manuscript have certified that they comply with the principles of ethical publishing in the International Journal of Cardiology.

\section{References}

[1] Jessup M, Brozena S. Medical progress: heart failure. N Engl J Med 2003;348: 2007-18.

[2] Nieminen MS, Brutsaert D, Dickstein K, et al. EuroHeart Failure Survey II (EHFS II): a survey on hospitalized acute heart failure patients: description of population. Eur Heart J 2006;27:2725-36.

[3] Cleland JGF, Swedberg K, Follath F, et al. The EuroHeart Failure survey programme a survey on the quality of care among patients with heart failure in Europe part 1: patient characteristics and diagnosis. Eur Heart J 2003;24:442-63.

[4] Krumholz HM, Parent EM, Tu N, et al. Readmission after hospitalization for congestive heart failure among Medicare beneficiaries. Arch Intern Med 1997;157: 99-104.

[5] Ronco C, McCullough P, Anker SD, et al. Cardio-renal syndromes: report from the consensus conference of the Acute Dialysis Quality Initiative. Eur Heart J 2010;31: 703-11.

[6] Krumholz HM, Chen YT, Vaccarino V, et al. Correlates and impact on outcomes of worsening renal function in patients $\geq 65$ years of age with heart failure. Am J Cardiol 2000;85:1110-3. 
[7] Forman DE, Butler J, Wang Y, et al. Incidence, predictors at admission, and impact of worsening renal function among patients hospitalized with heart failure. J Am Coll Cardiol 2004;43:61-7.

[8] Gottlieb SS, Abraham W, Butler J, et al. The prognostic importance of different definitions of worsening renal function in congestive heart failure. J Cardiac Fail 2002;8:136-41.

[9] Cowie MR, Komajda M, Murray-Thomas T, Underwood J, Ticho B; on behalf of the POSH investigators. Prevalence and impact of worsening renal function in patients hospitalized with decompensated heart failure: results of the prospective outcomes study in heart failure (POSH). Eur Heart J 2006;27:1216-22.

[10] Campbell CY, Clarke W, Park H, Haq N, Barone BB, Brotman DJ. Usefulness of cystatin $\mathrm{C}$ and prognosis following admission for acute heart failure. Am J Cardiol 2009;104:389-92.

[11] Manzano-Fernández S, Januzzi JL, Boronat-Garcia M, et al. $\beta$-Trace protein and cystatin $\mathrm{C}$ as predictors of long-term outcomes in patients with acute heart failure. J Am Coll Cardiol 2011;57:849-58.

[12] Lassus J, Harjola VP, Sund R, et al. Prognostic value of cystatin C in acute heart failure in relation to other markers of renal function and NT-proBNP. Eur Heart J 2007:28:1841-7.

[13] Xu S, Venge P. Lipocalins as biochemical markers of disease. Biochim Biophys Acta 2000;1482:298-307.

[14] Mori K, Lee HT, Rapoport D, et al. Endocytic delivery of lipocalin-siderophore-iron complex rescues the kidney from ischemia-reperfusion injury. J Clin Invest 2005;115:610-21.

[15] Schmidt-Ott KM, Mori K, Li JY, et al. Dual action of neutrophil gelatinaseassociated lipocalin. J Am Soc Nephrol 2007;18:407-13.

[16] Mori K, Nakao K. Neutrophil gelatinase-associated lipocalin as the real-time indicator of active kidney damage. Kidney Int 2007;71:967-70.

[17] Haase-Fielitz A, Bellomo R, Devarajan P, et al. The predictive performance of plasma neutrophil gelatinase-associated lipocalin (NGAL) increases with grade of acute kidney injury. Nephrol Dial Transplant 2009;24:3349-54.

[18] Mishra J, Dent C, Tarabishi R, et al. Neutrophil gelatinase-associated lipocalin (NGAL) as a biomarker for acute renal injury after cardiac surgery. Lancet 2005;365:1231-8.

[19] Wagener G, Gubitosa G, Wang S, Borregaard N, Kim M, Lee HT. Urinary neutrophil gelatinase-associated lipocalin and acute kidney injury after cardiac surgery. Am J Kidney Dis 2008:52:425-33.

[20] Haase M, Bellomo R, Devarajan P, Schlattmann P, Haase-Fielitz A; for the NGAL Meta-analysis Investigator Group. Accuracy of neutrophil gelatinase-associated lipocalin (NGAL) in diagnosis and prognosis in acute kidney injury: a systematic review and meta-analysis. Am J Kidney Dis 2009;54:1012-24.

[21] Alvelos M, Pimentel R, Pinho E, et al. Neutrophil gelatinase-associated lipocalin in the diagnosis of type 1 cardio-renal syndrome in the general ward. Clin J Am Soc Nephrol 2011;6:476-81.

[22] Ding H, He Y, Li K, et al. Urinary neutrophil gelatinase-associated lipocalin (NGAL) is an early biomarker for renal tubulointerstitial injury in IgA nephropathy. Clin Immunol 2007; 123:227-34.

[23] Liangos O, Perianayagam MC, Vaidya VS, et al. Urinary N-acetyl-beta-(D)glucosaminidase activity and kidney injury molecule-1 level are associated with adverse outcomes in acute renal failure. J Am Soc Nephrol 2007;18:904-12.
[24] Machiguchi T, Yoshida H, Yonemoto S, et al. Does circulating erythropoietin reflect progression of IgA nephropathy? Comparison with urinary N-acetyl-beta-Dglucosaminidase. Nephrol Dial Transplant 1999;14:635-40.

[25] Damman K, Van Veldhuisen DJ, Navis G, et al. Tubular damage in chronic systolic heart failure is associated with reduced survival independent of glomerular filtration rate. Heart 2010;96:1297-302.

[26] Bolignano D, Basile G, Parisi P, Coppolino G, Nicocia G, Buemi M. Increased plasma neutrophil gelatinase-associated lipocalin levels predict mortality in elderly patients with chronic heart failure. Rejuvenation Res 2009;12:7-14.

[27] Levey AS, Bosch JP, Lewis JB, Greene T, Rogers N, Roth D. A more accurate method to estimate glomerular filtration rate from serum creatinine: a new prediction equation. Modification of Diet in Renal Disease Study Group. Ann Intern Med 1999; $130: 461-70$

[28] O'Meara E, Chong KS, Gardner RS, Jardine AG, Neilly JB, McDonagh TA. The Modification of Diet in Renal Disease (MDRD) equations provide valid estimations of glomerular filtration rates in patients with advanced heart failure. Eur J Heart Fail 2006;8:63-7.

[29] Smilde TDJ, van Veldhuisen DJ, Navis G, Voors AA, Hillege HL. Drawbacks and prognostic value of formulas estimating renal function in patients with chronic heart failure and systolic dysfunction. Circulation 2006;114:1572-80.

[30] Nohria A, Hasselblad V, Stebbins A, et al. Cardiorenal interactions: insights from the ESCAPE trial. J Am Coll Cardiol 2008;51:1268-74.

[31] Bolignano D, Lacquaniti A, Coppolino G, et al. Neutrophil gelatinase-associated lipocalin (NGAL) and progression of chronic kidney disease. Clin J Am Soc Nephrol 2009;4:337-44.

[32] Bolignano D, Coppolino G, Lacquaniti A, Nicocia G, Buemi M. Pathological and prognostic value of urinary neutrophil gelatinase-associated lipocalin (NGAL) in macroproteinuric patients with worsening renal function. Kidney Blood Press Res 2008;31:274-9.

[33] Smith GL, Lichtman JH, Bracken MB, et al. Renal impairment and outcomes in heart failure: systematic review and meta-analysis. J Am Coll Cardiol 2006;47:1987-96.

[34] Damman K, Navis G, Voors AA, et al. Worsening renal function and prognosis in heart failure: systematic review and meta-analysis. J Cardiac Fail 2007;13: 599-608.

[35] Shlipak MG, Sarnak MJ, Katz R, et al. Cystatin-C and mortality in elderly persons with heart failure. J Am Coll Cardiol 2005;45:268-71.

[36] Djousse L, Kurth T, Gaziano JM. Cystatin C and risk of heart failure in the Physicians' Health Study (PHS). Am Heart J 2008;155:82-6.

[37] Alehagen U, Dahlstrom U, Lindahl TL. Cystatin C and NT-proBNP, a powerful combination of biomarkers for predicting cardiovascular mortality in elderly patients with heart failure: results from a 10-year study in primary care. Eur J Heart 2009;11: 354-60.

[38] Naruse H, Ishii J, Kawai T, et al. Cystatin C in acute heart failure without advanced renal impairment. Am J Med 2009;122:566-73.

[39] Carrasco-Sánchez FJ, Galisteo-Almeda L, Páez-Rubio I, et al. Prognostic value of cystatin $C$ on admission in heart failure with preserved ejection fraction. J Cardiac Fail 2011;17:31-8. 